\section{CAN WE INVOLVE ADOLESCENTS IN HIV VACCINE TRIALS - CAN WE AFFORD NOT TO?}

More than half of the world's population is under 25 years of age - the largest population of young people the world has ever seen. In South Africa, 54\% of the population is under 24 years of age. All are at risk for HIV infection.

UNAIDS reports that in 2004 there were 14000 new infections daily. Almost 100\% of these were in the developing world, 1200 were in 14 - 25-year-olds, and half of these were in females. Two-thirds of the infections in this age group occurred in sub-Saharan Africa. An HIV prevalence survey carried out in nearly 12000 young South Africans and recently reported in AIDS showed a prevalence rate of $2.3 \%$ in boys and $4.1 \%$ in girls at age 15 , increasing steadily to $5.9 \%$ in boys and a staggering $31.2 \%$ in girls by age $21 .{ }^{1}$

So what are the risk factors for infection in these young people? Almost universally sex, with numbers most driven by heterosexual sex in young women in sub-Saharan Africa. Other risk factors include homosexual sex, while intravenous drug use is an increasing problem, especially in Eastern Europe and Central Asia.

Women comprise three-quarters of infections among young heterosexual people in Africa. This is because HIV transmission is more efficient from men to women. A number of factors contribute to this, e.g. young women are having sex with older men (trans-generational), putting them at more risk of encountering positive partners, but education and coercion also play a part. Biological reasons include cervical ectopy, hormonal influences, pregnancy-related infection and concomitant sexually transmitted infections. ${ }^{2}$

In addition, youth may be vulnerable in their decision making around sexual practices owing to inexperience, a transitional emotional state, drug and other substance abuse, marginalised activities, economic forces and coercion. All of these factors make heterosexual youth in resource-poor settings, intravenous drug-using youth in other communities and young men who have sex with men in many countries at increased risk of HIV infection.

Sexual behaviour surveys have been carried out extensively in South Africa, with at least 35 published articles on HIV knowledge and 75 papers on sexual behaviour and risk. These studies confirm that South African youth have an early sexual debut $(<15$ years) and indulge in relatively high-risk behaviour. This has made AIDS the leading cause of death.

Yet research in adolescents is fraught: they have not reached the age of legal majority, they are undergoing hormonal upheavals, and they are in a transitional phase of social development. Anyone who has had teenagers in their household will know the quest for identity, autonomy and control, the distrust of hierarchy and establishment, the strong effects of peer pressure and social norms, and a strong element of invincibility or risk taking. Participation in research is complicated by the requirement for consent from a legal guardian, the need to accommodate school hours and homework, mobility in terms of future plans, and reliance on public transport to get to clinic appointments. Information on sexual risk can be difficult to get and may have questionable reliability. While adolescents are unsophisticated and in their formal operational phase of data processing, they are trainable. $^{4}$

Why then even consider adolescents for clinical vaccine trials? The argument comes down to a risk/benefit one. On the one hand we have the problem of conducting 'non-therapeutic' research in vulnerable minors, but on the other we have a lifethreatening pandemic of unprecedented proportions that is especially targeting youth in developing countries. There is scientific justification for the argument that immune responses may be adversely affected by the huge hormonal shifts occurring at this age, but other vaccine trials have indicated that age may play a positive part in responses, e.g. hepatitis $B$ (better responses with half the dose used for 11 - 19-year-olds compared with 20 - 30-year olds), while in HPV trials the geometric mean titres were 2-fold higher in the 10 - 15-year age group compared with $16-23$ years.

Another reason to involve adolescents in vaccine clinical testing at this stage is that regulatory bodies will almost certainly require adolescent experience for licensure and we certainly don't want to lag in implementation of a vaccine in teenagers should one look promising. Finally, the actual rollout and distribution of an effective vaccine will be a monumental task worldwide and we should be prepared for this. Involvement of adolescents now may prepare the way. The early age of sexual debut and risk for HIV acquisition indicates that our window of opportunity to implement a preventive vaccine occurs before the age of 14 years. Our feasibility studies in Cape Town have shown that there is adolescent interest in participation and that motivation is largely altruistic. Since the adolescent HIV epidemic is such a serious problem for South Africa, and we have the expertise and apparently the enthusiasm here to overcome the obstacles, it seems to me that South Africa must lead the way in getting adolescents involved as soon as possible.

This article was derived from presentations delivered by Jared Baeten, University of Washington, Seattle, and Linda-Gail Bekker at the 2005 AIDS Vaccine Conference, Montreal.

\section{LINDA-GAIL BEKKER}

Managing Editor

1. Pettifor, et al. AIDS 2005; 19: 1525-1534.

2. Plourde, et al. J Inf Dis 1994; 170: 313-317.

4. L Swartz, et al. JAIPAC 2005 (in press).

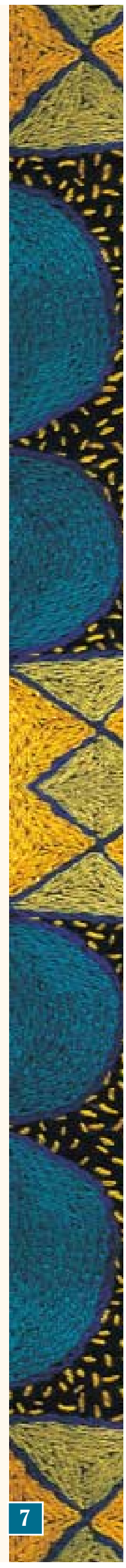

